\title{
Article \\ Forward Left Ventricular Ejection Fraction as a Predictor of Postoperative Left Ventricular Dysfunction in Patients with Degenerative Mitral Regurgitation
}

\author{
Juyoun Kim, Jae-Sik Nam $\mathbb{D}^{\mathbb{D}}$, Youngdo Kim $\mathbb{D}^{\mathbb{C}}$, Ji-Hyun Chin *(D) and In-Cheol Choi
}

check for

updates

Citation: Kim, J.; Nam, J.-S.; Kim, Y.; Chin, J.-H.; Choi, I.-C. Forward Left Ventricular Ejection Fraction as a Predictor of Postoperative Left Ventricular Dysfunction in Patients with Degenerative Mitral Regurgitation. J. Clin. Med. 2021, 10, 3013. https://doi.org/10.3390/ jcm10143013

Academic Editor: Pierluigi Omedè

Received: 31 May 2021

Accepted: 5 July 2021

Published: 6 July 2021

Publisher's Note: MDPI stays neutral with regard to jurisdictional claims in published maps and institutional affiliations.

Copyright: (c) 2021 by the authors. Licensee MDPI, Basel, Switzerland. This article is an open access article distributed under the terms and conditions of the Creative Commons Attribution (CC BY) license (https:// creativecommons.org/licenses/by/ $4.0 /$ )
Department of Anesthesiology and Pain Medicine, Asan Medical Center, University of Ulsan College of Medicine, Seoul 05505, Korea; juyoun1012@naver.com (J.K.); jaesik_nam@naver.com (J.-S.N.); midorimd@naver.com (Y.K.); icchoi@amc.seoul.kr (I.-C.C.)

* Correspondence: cjh@amc.seoul.kr; Tel.: +82-2-3010-5632; Fax: +82-2-3010-6790

\begin{abstract}
Background: Left ventricular dysfunction (LVD) can occur immediately after mitral valve repair (MVr) for degenerative mitral regurgitation (DMR) in some patients with normal preoperative left ventricular ejection fraction (LVEF). This study investigated whether forward LVEF, calculated as left ventricular outflow tract stroke volume divided by left ventricular end-diastolic volume, could predict LVD immediately after MVr in patients with DMR and normal LVEF. Methods: Echocardiographic and clinical data were retrospectively evaluated in 234 patients with DMR $\geq$ moderate and preoperative LVEF $\geq 60 \%$. LVD and non-LVD were defined as LVEF $<50 \%$ and $\geq 50 \%$, respectively, as measured by echocardiography after MVr and before discharge. Results: Of the 234 patients, $52(22.2 \%)$ developed LVD at median three days (interquartile range: 3-4 days). Preoperative forward LVEF in the LVD and non-LVD groups were 24.0\% (18.9-29.5\%) and 33.2\% (26.4-39.4\%), respectively $(p<0.001)$. Receiver operating characteristic (ROC) analyses showed that forward LVEF was predictive of LVD, with an area under the ROC curve of 0.79 (95\% confidence interval: $0.73-0.86$ ), and an optimal cut-off was $31.8 \%$ (sensitivity: $88.5 \%$, specificity: $58.2 \%$, positive predictive value: $37.7 \%$, and negative predictive value: $94.6 \%$ ). Preoperative forward LVEF significantly correlated with preoperative mitral regurgitant volume (correlation coefficient $[C C]=-0.86, p<0.001$ ) and regurgitant fraction (CC $=-0.98, p<0.001)$, but not with preoperative LVEF $(C C=0.112, p=0.088)$. Conclusion: Preoperative forward LVEF could be useful in predicting postoperative LVD immediately after $\mathrm{MVr}$ in patients with DMR and normal LVEF, with an optimal cut-off of $31.8 \%$.
\end{abstract}

Keywords: degenerative mitral regurgitation; forward left ventricular ejection fraction; mitral valve repair; postoperative left ventricular dysfunction

\section{Introduction}

Degenerative mitral regurgitation (DMR) is the second most common valvular heart disease [1]. Mitral valve surgery is recommended before the onset of left ventricular (LV) dysfunction (LVD) [2], primarily because preoperative LVD has been associated with unfavorable outcomes [3-5], and early mitral valve surgery was found to be associated with a greater long-term survival benefit and a lower risk of heart failure than medical treatment [6].

LVD has been observed after mitral valve surgery for DMR, even in patients with normal preoperative LV function, with studies attempting to identify preoperative echocardiographic parameters that are associated with postoperative LVD [7-12]. Studies assessing factors that correlate with postoperative LVD occurring immediately after mitral valve surgery have identified that LV ejection fraction (LVEF), LV end-systolic diameter (LVESD), preoperative right ventricular systolic pressure and global longitudinal strain may be associated with immediately postoperative LVD [7-13]. A previous report demonstrated that only about one-third of patients with LVD immediately after surgery experienced recovery of LV function during long-term follow-up [7]. Therefore, identification of factors 
predictive of immediately postoperative LVD may be clinically significant, in that it may help to determine patients requiring early surgery to minimize LVD occurrence.

Forward LVEF, calculated as forward stroke volume (SV) divided by LV end-diastolic volume (LVEDV), may helpful in stratifying the long-term risk in patients with $\geq$ mild MR [14]. Few studies to date have evaluated the ability of forward LVEF to predict short-term risk, especially in terms of LVD after mitral valve surgery. The present study investigated whether forward LVEF could predict LVD immediately after mitral valve repair (MVr) in patients with DMR and normal preoperative LVEF.

\section{Materials and Methods}

\subsection{Study Population}

This retrospective observational study included patients who underwent MVr for DMR with grade $\geq$ moderate, at Asan Medical Center (Seoul, Korea) from January 2010 to December 2018. Patients were excluded if they had preoperative LVEF $<60 \%$ or coronary artery disease; if forward LVEF could not be calculated because of limited data; if they had undergone redo mitral valve surgery; or if immediately postoperative echocardiography showed remnant MR with grade $\geq$ moderate. The research protocol was approved by our Institutional Review Board (AMC IRB 2020-1918), which waived the requirement for written informed consent because of the retrospective nature of the study. Data were acquired from a retrospective review of electronic medical records.

\subsection{Echocardiographic Data}

All patients underwent transthoracic echocardiographic examination before and after MVr. Our institution followed the standards and techniques recommended by the American Society of Echocardiography for measuring MR severity $[15,16]$. Two-dimensional echocardiography and Doppler color flow imaging were performed in all patients using a Hewlett-Packard Sonos 2500, 5500, or 7500 imaging system (Hewlett-Packard, Andover, MA, USA) and a VIVID 7 or E9 ultrasound system (General Electric Healthcare, Little Chalfont, UK) with a $2.5 \mathrm{MHz}$ probe. The left ventricular end-diastolic diameter and LV end-systolic diameter (LVESD) were measured from parasternal M-mode acquisitions, and the LV end-systolic volume (LVESV) and LVEDV were measured using the biplane Simpson method. LVEF was calculated from the measured LVESV and LVEDV. Measurements were averaged over three to five cardiac cycles for patients with atrial fibrillation.

Comprehensive echocardiographic evaluation of mitral regurgitation was performed using an integrated approach including 2-dimensional, Doppler, and color flow imaging. The proximal isovelocity surface area (PISA) was determined by measuring the proximal flow convergence by lowering the imaging depth and reducing the Nyquist limit at midsystole. Various views were evaluated for optimal visualization of the PISA. Baseline shift was used to adjust the aliasing velocity to about $40 \mathrm{~cm} / \mathrm{s}$.

Forward LVEF was calculated using the following equation: forward LVEF $=100 \times$ forward SV/LVEDV, where the forward SV was measured by pulsed wave Doppler in the $\mathrm{LV}$ outflow tract [14]. To determine intra-observer and inter-observer variability, a random sample of $25 \%$ of left ventricular outflow tract (LVOT) recordings was submitted twice to the first investigator and once to a second investigator. The inter-observer variability was calculated as the mean absolute difference between the two readings from the first and the second investigator divided by their mean. Similarly, the intra-observer variability was calculated as the mean absolute difference between the two readings from the first investigator divided by their mean.

In addition, we calculated the midwall fractional shortening (mFS) to assess LV contractility. The actual midwall fractional shortening (mFS) was determined using the two-shell method of Shimizu et al. [17]. Circumferential end-systolic stress (cESS), a measure of ventricular afterload, was calculated at the midwall according to the method of Gaasch et al. [18]. Thereafter, the predicted $\mathrm{mFS}$ was determined for any given cESS using the regression equation derived from a healthy population [19]. To minimize after- 
load dependence, stress-corrected mFS (sc-mFS) was calculated as the ratio of actual to predicted mFS [19].

Preoperative echocardiographic data were those determined closest to the day of surgery, and postoperative echocardiographic data were those determined before discharge without significant inotropic support or a mechanical assist device. The postoperative period was defined as after MVr and before hospital discharge.

\subsection{Statistical Analysis}

Continuous data are presented as mean \pm standard deviation (SD) or as median (interquartile range [IQR]), and categorical data are presented as frequencies (percentages). The LVD and non-LVD groups were defined as patients with LVEF $<50 \%$ and $\geq 50 \%$, respectively, as measured by echocardiography after MVr and before discharge. Continuous data in the LVD and non-LVD groups were compared by Student's t-test or Mann- Whitney U test, and categorical data by Chi-square test or Fisher's exact test, as appropriate. Within group echocardiographic parameters measured preoperatively and immediately postoperatively were compared by paired t-test or Wilcoxon signed rank test, as appropriate.

Receiver operating characteristic (ROC) curve analyses were performed to determine the performance of individual parameters for predicting LVD. The optimal cut-off on ROC curves was defined as the value based on Youden's Index, which was calculated as maximum (sensitivity + specificity -1 ). Correlations of forward LVEF, mitral regurgitant volume (RVol), mitral regurgitant fraction $(\mathrm{RF})$, and LVEF were calculated using Pearson or Spearman correlation analyses. $p<0.05$ was considered statistically significant. We conducted analyses using the SigmaPlot 13.0 (Systat Software Inc., San Jose, CA, USA).

\section{Results}

During the study period, 706 patients underwent MVr for DMR. Of these, there were 472 patients who did not satisfy the inclusion criteria as follows: 30 with preoperative LVEF $<60 \%$, 9 with coronary artery disease, 427 who did not have available data for calculating forward LVEF, 1 who underwent redo mitral valve surgery, 4 with MR $\geq$ moderate at immediately postoperative echocardiography, and 1 with inotropic support during immediately postoperative echocardiographic examination. Thus, the remaining 234 patients were evaluated.

Patients underwent immediate postoperative echocardiography three (3-4) days after MVr. Of 234 patients, 52 (22.2\%) experienced immediately postoperative LVD. The preoperative demographic characteristics, comorbidities, and medications are presented in Table 1. Chronic kidney disease and atrial fibrillation were more prevalent in the LVD than in the non-LVD group. By contrast, age, the proportion of male, Euroscore II, preoperative medications, and cardiopulmonary bypass and aortic cross clamping times were similar in the two groups.

A comparison of preoperative echocardiographic parameters in the two groups showed that preoperative LVEF, forward SV, and forward LVEF were lower in the LVD than in the non-LVD group, whereas preoperative LVESD, LVEDV, LV mass index, RVol and RF were higher in the LVD than in the non-LVD group (Table 2).

The inter-observer and intra-observer variabilities for LVOT measurement were $5.0 \pm 2.9 \%$ and $4.3 \pm 2.7 \%$, respectively.

Differences in echocardiographic parameters measured preoperatively and immediately postoperatively were also assessed in the two groups. LVEDV, LVEF, $\mathrm{mFS}$, and sc-mFS were lower postoperatively than preoperatively in both groups, whereas cESS was lower postoperatively only in the non-LVD group (Table 3). Between-group comparisons of postoperative parameters showed that postoperative LVEDV was lower, whereas postoperative sc-mFS was higher, in the non-LVD than in the LVD group (Table 3). 
Table 1. Baseline characteristics.

\begin{tabular}{|c|c|c|c|c|}
\hline & Total & LVD & Non-LVD & $p$ \\
\hline \multicolumn{5}{|c|}{ Demographics } \\
\hline Age (years) & $51.8 \pm 11.8$ & $52.2 \pm 10.8$ & $51.7 \pm 12.1$ & 0.781 \\
\hline Male & $165(70.5)$ & $37(71.2)$ & $127(69.8)$ & 1.0 \\
\hline BMI $\left(\mathrm{kg} / \mathrm{m}^{2}\right)$ & $24.7(22.5-26.5)$ & $24.3(21.6-25.9)$ & $24.7(22.7-26.7)$ & 0.113 \\
\hline \multicolumn{5}{|c|}{ Comorbidities } \\
\hline $\mathrm{DM}$ & $41(17.5)$ & $12(23.1)$ & $29(15.9)$ & 0.3 \\
\hline $\mathrm{HTN}$ & $93(39.7)$ & $20(38.5)$ & $73(40.1)$ & 0.873 \\
\hline CVA & $5(2.1)$ & $0(0)$ & $5(2.7)$ & 0.589 \\
\hline PVD & $2(0.9)$ & $0(0)$ & $2(1.1)$ & 1.0 \\
\hline COPD & $7(3.0)$ & $0(0)$ & $7(3.8)$ & 0.353 \\
\hline CKD & $6(2.6)$ & $3(5.8)$ & $3(1.6)$ & 0.005 \\
\hline A-fib & $26(11.1)$ & $11(21.2)$ & $15(8.2)$ & 0.021 \\
\hline Euroscore II & $0.7(0.5-1.0)$ & $0.7(0.6-1.0)$ & $0.7(0.5-1.0)$ & 0.445 \\
\hline \multicolumn{5}{|c|}{ Preoperative Medication } \\
\hline $\mathrm{ACEI} / \mathrm{ARB}$ & $112(47.9)$ & $26(50.0)$ & $86(47.3)$ & 0.755 \\
\hline$\beta$-blocker & $68(29.1)$ & $16(30.8)$ & $52(28.6)$ & 0.733 \\
\hline CCB & $52(22.2)$ & $13(25.0)$ & $39(21.4)$ & 0.575 \\
\hline Digoxin & $15(6.4)$ & $3(5.8)$ & $12(6.6)$ & 1.0 \\
\hline Diuretics & 107 (45.7) & $25(48.1)$ & $82(45.1)$ & 0.753 \\
\hline \multicolumn{5}{|c|}{ Intraoperative Data } \\
\hline Op time (mins) & $\begin{array}{c}275.0 \\
(237.0-323.3)\end{array}$ & $\begin{array}{c}256.5 \\
(233.3-305.0)\end{array}$ & $\begin{array}{c}280.0 \\
(237.0-325.0)\end{array}$ & 0.187 \\
\hline CPB time (mins) & $\begin{array}{c}140.0 \\
(115.8-170.0)\end{array}$ & $\begin{array}{c}144.5 \\
(113.0-167.5)\end{array}$ & $\begin{array}{c}138.0 \\
(115.8-170.5)\end{array}$ & 0.679 \\
\hline ACC time (mins) & $77.5(64.0-99.0)$ & $84.0(71.3-107.0)$ & $77.0(63.0-96.0)$ & 0.093 \\
\hline Colloid (L) & $0.5(0.3-0.5)$ & $0.5(0.4-0.5)$ & $0.5(0.3-0.5)$ & 0.095 \\
\hline Crystalloid (L) & $1.5(1.2-1.8)$ & $1.5(1.0-1.6)$ & $1.5(1.2-1.8)$ & 0.073 \\
\hline
\end{tabular}

Data are presented as mean \pm SD, median (interquartile range), or number (percentage). The LVD and nonLVD groups were defined as patients with left ventricular ejection fraction $<50 \%$ and $\geq 50 \%$, respectively, as measured by echocardiography after mitral valve repair and before discharge. BMI = body mass index; $\mathrm{DM}=$ diabetes mellitus; HTN = hypertension; CVA = cerebrovascular accident; PVD = peripheral vascular disease; $\mathrm{COPD}=$ chronic obstructive pulmonary disease; $\mathrm{CKD}=$ chronic kidney disease; $\mathrm{A}$-fib = atrial fibrillation $\mathrm{ACEI}=$ angiotensin converting enzyme inhibitor; $\mathrm{ARB}=$ angiotensin receptor blocker; $\mathrm{CCB}=$ calcium channel blocker; $\mathrm{CPB}=$ cardiopulmonary bypass; $\mathrm{ACC}=$ aorta cross clamping.

ROC analyses showed that forward LVEF was predictive of LVD, with an area under the ROC curve of 0.79 ( $95 \%$ confidence interval: $0.73-0.86$ ). The optimal cut-off of $31.8 \%$ had a sensitivity of $88.5 \%$, a specificity of $58.2 \%$, a positive predictive value (PPV) of $37.7 \%$, and a negative predictive value (NPV) of $94.6 \%$ (Figure 1, Table 4). The area under the ROC curve of LVESD was 0.75 (95\% CI: 0.67-0.83), with an optimal cut-off of $38 \mathrm{~mm}$ (sensitivity of $65.4 \%$, specificity of $74.2 \%$, PPV of $42.2 \%$, and NPV of $88.2 \%$ ) (Table 4 ).

In addition, preoperative forward LVEF correlated significantly with preoperative $\mathrm{RVol}$ (correlation coefficient $[\mathrm{CC}]=-0.86, p<0.001)$ and $\mathrm{RF}(\mathrm{CC}=-0.98, p<0.001)$ (Figure 2), but not with preoperative LVEF (CC $=0.112, p=0.088)$ and peak systolic velocity of mitral annulus (CC $=0.04, p=0.529)$.

There were 62 patients (16 in the LVD group and 46 in the non-LVD group) who had available LVEF data at six months after MVr. LVEFs at six months were 55.5\% (51.3-59.8\%) and $60.5 \%(57.8-65.0 \%)$ in the LVD group and the non-LVD group, respectively $(p<0.001)$.

Between 6 and 18 months after MVr, LVEF data were available for 212 of the 234 included patients. Of these 212 patients, three $(1.4 \%)$, all in the LVD group, showed LVEF $<50 \%$ at $6-18$ months. 
Table 2. Preoperative echocardiographic findings.

\begin{tabular}{ccccc}
\hline & Total & LVD & Non-LVD & $p$ \\
\hline LVEF & $68.0(65.0-71.0)$ & $66.5(64.0-69.0)$ & $68.0(65.0-72.0)$ & 0.020 \\
LVESD & $36.7 \pm 5.2$ & $40.3 \pm 4.9$ & $35.6 \pm 4.8$ & $<0.001$ \\
LVEDD & $59.6 \pm 5.5$ & $63.8 \pm 4.5$ & $58.4 \pm 5.1$ & $<0.001$ \\
LVESV & $56.0(43.0-68.0)$ & $70.5(58.0-80.0)$ & $51.5(41.0-62.3)$ & $<0.001$ \\
LVEDV & $172.0(141.8-209.3)$ & $210.5(184.3-238.5)$ & $163.0(137.0-197.0)$ & $<0.001$ \\
RWT & $0.32(0.29-0.35)$ & $0.30(0.27-0.32)$ & $0.33(0.29-0.36)$ & $<0.001$ \\
LVMI & $129.3(112.3-151.6)$ & $149.6(133.3-168.5)$ & $123.5(108.5-141.9)$ & $<0.001$ \\
LAD & $47.0(43.0-53.0)$ & $49.5(46.3-55.8)$ & $46.0(43.0-52.0)$ & $<0.001$ \\
PG & $29.0(23.0-36.0)$ & $29.0(27.0-38.0)$ & $27.0(23.0-36.0)$ & 0.104 \\
E/A & $2.0(1.5-2.5)$ & $2.2(1.7-2.5)$ & $1.9(1.4-2.6)$ & 0.303 \\
S' & $7.9(7.0-8.7)$ & $7.9(7.1-8.9)$ & $7.9(7.0-8.7)$ & 0.568 \\
E' & $8.0(6.4-9.9)$ & $8.3(6.3-9.6)$ & $8.0(6.5-10.0)$ & 0.897 \\
A' & $7.5(6.2-8.9)$ & $7.1 \pm 1.7$ & $7.6 \pm 1.9$ & 0.098 \\
E/E' & $14.0(11.0-19.0)$ & $15.5(11.3-19.8)$ & $14.0(11.0-19.0)$ & 0.234 \\
cESS & $135.8(113.2-161.3)$ & $154.0(129.8-170.5)$ & $131.9(112.2-157.9)$ & 0.006 \\
mFS & $20.3(18.6-21.9)$ & $20.2(18.7-21.9)$ & $20.3(18.5-21.9)$ & 0.843 \\
sc-mFS & $118.9(109.5-130.4)$ & $123.7(111.9-131.3)$ & $118.2(108.3-130.4)$ & 0.114 \\
Forward SV & $53.1(45.0-60.6)$ & $50.1(43.3-59.2)$ & $54.0(46.0-61.8)$ & 0.074 \\
Forward LVEF & $31.4(24.9-37.4)$ & $24.0(18.9-29.5)$ & $33.2(26.8-39.4)$ & $<0.001$ \\
EROA & $0.8(0.6-1.1)$ & $1.0(0.8-1.3)$ & $0.8(0.6-1.1)$ & $<0.001$ \\
RVol & $64.5(44.0-86.4)$ & $85.7(69.6-110.8)$ & $57.5(39.3-78.0)$ & $<0.001$ \\
RF & $54.0(45.4-62.7)$ & $62.7(54.4-70.1)$ & $51.8(41.5-60.0)$ & $<0.001$
\end{tabular}

Data are presented as mean \pm SD or median (interquartile range). The LVD and non-LVD groups were defined as patients with $\mathrm{LVEF}<50 \%$ and $\geq 50 \%$, respectively, as measured by echocardiography after mitral valve repair and before discharge. $\mathrm{LVEF}=$ left ventricular ejection fraction; LVESD = left ventricular end-systolic diameter LVEDD = left ventricular end-diastolic diameter; LVESV = left ventricular end-systolic volume; LVEDV = left ventricular end-diastolic volume; RWT = relative wall thickness; LVMI = left ventricular mass index; LAD = left atrial diameter; $\mathrm{PG}_{\mathrm{TR}}=$ pressure gradient calculated from peak tricuspid regurgitation; $\mathrm{E} / \mathrm{A}=$ ratio of peak early and late diastolic velocity of mitral inflow; $S^{\prime}=$ peak systolic velocity of mitral annulus; $E^{\prime}=$ peak early diastolic velocity of mitral annulus; $\mathrm{A}^{\prime}$ = peak late diastolic velocity of mitral annulus; $\mathrm{E} / \mathrm{E}^{\prime}=$ ratio of peak early diastolic velocity of mitral inflow to mitral annulus early diastolic velocity; $\mathrm{cESS}=$ circumferential end-systolic stress; $\mathrm{mFS}$ = midwall fractional shortening; $\mathrm{sc}-\mathrm{mFS}=$ stress-corrected midwall fractional shortening; $\mathrm{SV}=$ stroke volume $\mathrm{EROA}=$ effective regurgitant orifice area; $\mathrm{RVol}=$ regurgitant volume of mitral regurgitation; $\mathrm{RF}=$ regurgitant fraction of mitral regurgitation.

Table 3. Differences in echocardiographic findings between preoperative and immediately after mitral valve repair.

\begin{tabular}{|c|c|c|c|c|c|c|c|c|}
\hline & & LVD & & & Non-LVD & & & \\
\hline & Pre & Post & $p^{*}$ & Pre & Post & $p^{*}$ & $p^{\dagger}$ & $p \ddagger$ \\
\hline $\begin{array}{l}\text { LVEDV } \\
\text { (preload) }\end{array}$ & $\begin{array}{c}210.5 \\
(184.3-238.5)\end{array}$ & $\begin{array}{c}141.5 \\
(119.3-187.3)\end{array}$ & $<0.001$ & $\begin{array}{c}163.0 \\
(137.0-197.0)\end{array}$ & $\begin{array}{c}114.0 \\
(95.8-137.0)\end{array}$ & $<0.001$ & $<0.001$ & $<0.001$ \\
\hline $\begin{array}{c}\text { cESS } \\
\text { (afterload) }\end{array}$ & $\begin{array}{c}154.0 \\
(129.8-170.5)\end{array}$ & $\begin{array}{c}151.3 \\
(133.4-186.7)\end{array}$ & 0.122 & $\begin{array}{c}131.9 \\
(112.2-157.9)\end{array}$ & $\begin{array}{c}120.8 \\
(101.9-138.5)\end{array}$ & $<0.001$ & 0.006 & $<0.001$ \\
\hline LVEF & $66.5(64.0-69.0)$ & $45.0(41.0-48.8)$ & $<0.001$ & $68.0(65.0-72.0)$ & $58.0(55.0-62.0)$ & $<0.001$ & 0.020 & $<0.001$ \\
\hline$S^{\prime}$ & $7.9(7.1-8.9)$ & $5.5(4.8-6.3)$ & $<0.001$ & $7.9(7.0-8.7)$ & $6.8(5.9-7.9)$ & $<0.001$ & 0.568 & $<0.001$ \\
\hline $\mathrm{mFS}$ & $20.2(18.7-21.9)$ & $13.4(12.1-15.3)$ & $<0.001$ & $20.3(18.5-21.9)$ & $16.0(14.4-17.5)$ & $<0.001$ & 0.843 & $<0.001$ \\
\hline sc-mFS & $\begin{array}{c}123.7 \\
(111.9-131.3)\end{array}$ & $82.5(72.3-92.0)$ & $<0.001$ & $\begin{array}{c}118.2 \\
(108.3-130.4)\end{array}$ & $\begin{array}{c}91.9 \\
(84.9-100.2)\end{array}$ & $<0.001$ & 0.114 & $<0.001$ \\
\hline
\end{tabular}

Data are presented as median (interquartile range). The LVD and non-LVD groups were defined as patients with LVEF $<50 \%$ and $\geq 50 \%$, respectively, as measured by echocardiography after mitral valve repair and before discharge. Pre $=$ preoperative; Post $=$ immediately postoperative; LVEDV = left ventricular end-diastolic volume; cESS = circumferential end-systolic stress; LVEF = left ventricular ejection fraction; $S^{\prime}=$ peak systolic velocity of mitral annulus; $\mathrm{mFS}=$ midwall fractional shortening; $\mathrm{sc}-\mathrm{mFS}=\mathrm{stress-corrected}$ midwall fractional shortening. ${ }^{*} p<0.05$ in comparison of parameters between preoperative and postoperative period in each group. ${ }^{\dagger} p<0.05$ in comparison of preoperative parameters between LVD and non-LVD groups. ${ }^{\ddagger} p<0.05$ in comparison of postoperative parameters between LVD and non-LVD groups. 
A

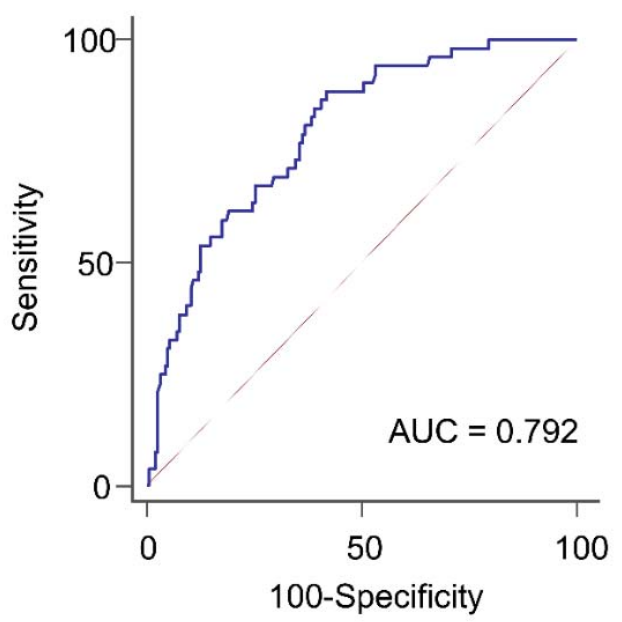

B

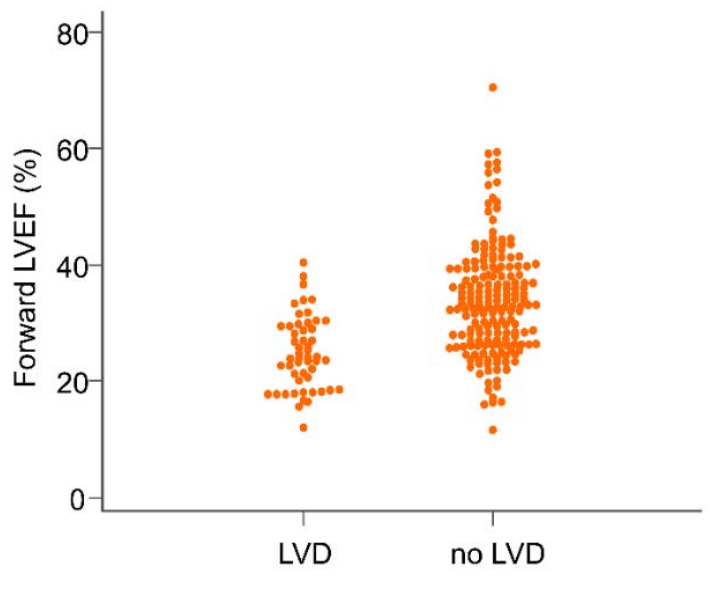

Figure 1. (A) Receiver operating characteristic curves demonstrating the performance of preoperative forward left ventricular ejection fraction (LVEF) to predict immediately postoperative left ventricular dysfunction (LVD), and (B) individual values of preoperative forward LVEF in the LVD and non-LVD group.

Table 4. Predictive ability of echocardiographic parameters for LVD immediately after MVr.

\begin{tabular}{ccccccc}
\hline & AUC (95\% CI) & Cut-off & Sen (\%) & Spe (\%) & PPV (\%) & NPV (\%) \\
\hline LVESD (mm) & $0.75(0.67-0.83)$ & 38.0 & 65.4 & 74.2 & 42.0 & 88.2 \\
Forward & $0.79(0.73-0.86)$ & 31.8 & 88.5 & 58.2 & 37.7 & 94.6 \\
LVEF (\%) & $0.78(0.71-0.84)$ & 73.5 & 73.1 & 70.3 & 41.3 & 90.1 \\
RVol (mL) & $0.77(0.70-0.84)$ & 60.6 & 65.4 & 78.6 & 46.6 & 88.8 \\
RF (\%) & $0.77(0.70-0.84)$ & 196.0 & 71.2 & 74.2 & 43.0 & 89.8 \\
LVEDV (mL) & 55.0 & 86.5 & 58.8 & 36.0 & 93.6 \\
LVESV (mL) & $0.78(0.72-0.85)$ &
\end{tabular}

$\mathrm{LVD}=$ left ventricular dysfunction; $\mathrm{MVr}=$ mitral valve repair; $\mathrm{AUC}=$ area under curve; $\mathrm{CI}=$ confidence interval Sen = sensitivity; Spe = specificity; $\mathrm{PPV}=$ positive predictive value; NPV = negative predictive value; $\mathrm{LVESD}=$ left ventricular end-systolic diameter; $\mathrm{LVEF}=$ left ventricular ejection fraction; $\mathrm{RVol}=$ regurgitant volume of mitral regurgitation; $\mathrm{RF}$ = regurgitant fraction of mitral regurgitation; LVEDV = left ventricular end-diastolic volume; LVESV = left ventricular end-systolic volume.

A

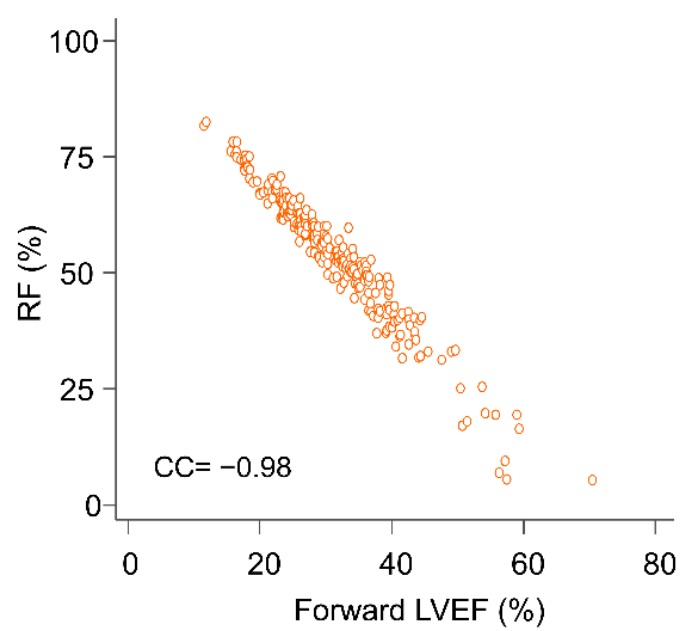

B

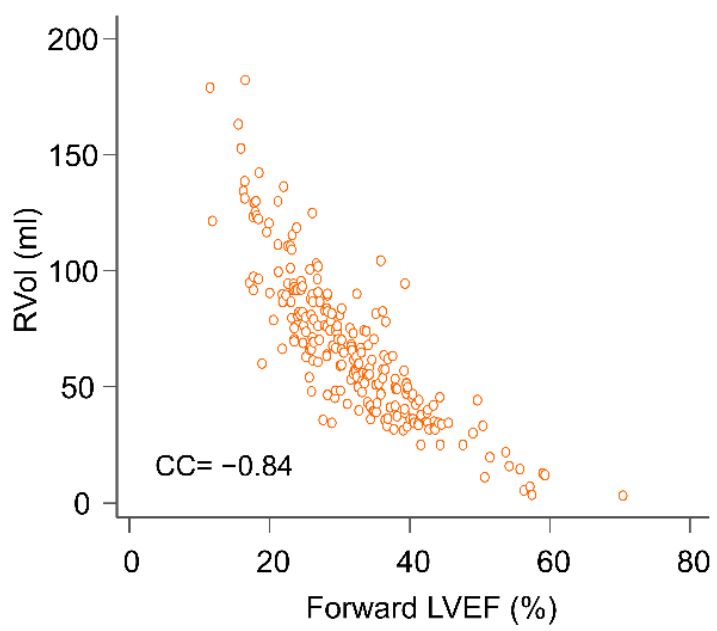

Figure 2. Correlations (A) between forward left ventricular ejection fraction (LVEF) and mitral regurgitant fraction (RF), and (B) between forward LVEF and mitral regurgitant volume (RVol). 


\section{Discussion}

The present study showed that preoperative forward LVEF could predict LVD immediately after $\mathrm{MVr}$, with an optimal cut-off of $31.8 \%$ in patients with DMR and preoperative $\mathrm{LVEF} \geq 60 \%$. Compared with preoperative LVESD, preoperative forward LVEF showed a similar AUC on ROC analysis, but a higher NPV for predicting postoperative LVD.

Although many previous studies have evaluated postoperative LVD after MVr, few studies have investigated postoperative LVD occurring immediately after mitral valve surgery $[7,11]$. High right ventricular systolic pressure and LVESD were found to be independently associated with an increased likelihood of postoperative LVEF $<40 \%$, with the latter being associated with increased mortality in patients with DMR and preoperative LVEF $\geq 60 \%$ who underwent $\mathrm{MVr}$ [7]. In addition, global longitudinal strain was found to be associated with postoperative LVEF reduction $>10 \%$ immediately after $\mathrm{MVr}$ for DMR [11].

Assessment of LV systolic function in asymptomatic patients with DMR is regarded as important, because mitral valve surgery is recommended before onset of LV systolic dysfunction [2]. Preoperative LVEF is regarded as indicative of LV systolic dysfunction, although LVEF could not be reflective of LV function because of a mechanism that compensated for volume overload of the left ventricle in patients with MR. Previous studies reported LV dysfunction present in patients with DMR and normal preoperative LVEF [20-24]. Marked myofibrillar degeneration and myocardial fibrosis, indicating LV contractile dysfunction, have been observed in patients with preoperative LVEF $\geq 60 \%$ before surgery. LV fibrosis was found to be more prevalent in patients with than without MV prolapse at all levels of MR fraction [20]. Moreover, diffuse interstitial fibrosis was a prevalent finding, occurring prior to any conventional class I indications for mitral valve surgery. This finding suggested that MR did not have a physiological compensatory phase and that volume overload may lead to pathological changes in LV structure and function at an earlier stage [21]. LV dysfunction may be masked by normal, even supra-normal, LVEF, with this latent LV dysfunction revealed in the absence of overload [14]. The actual stage of preoperative LV function may be reflected by immediately postoperative LVEF [12,14], as surgical correction results in the disappearance of the confounding effect of mitral regurgitant volume, although the effects of changes in preload and afterload cannot be completely excluded.

Forward LVEF was found to be independently associated with long-term outcome, composite of mitral valve surgery and death, in patients with $\geq$ mild MR, and may be superior to LVEF and LVESD in predicting outcomes [14]. That study also demonstrated that forward LVEF could predict postoperative LVD, with a cut-off value of $40 \%$ [14]. That study, however, did not specify the preoperative LVEF of studied patients and the timing of LVD occurrence. Another study reported that preoperative forward LVEF $<40 \%$ could predict LVD three months after surgery in a small number of patients undergoing mitral valve surgery [25]. In our study, forward LVEF could predict LVD immediately after $\mathrm{MVr}$, with an optimal cut-off of $31.8 \%$ in patients with DMR and preoperative LVEF $\geq 60 \%$. When compared with conventional parameters, the AUCs for preoperative forward LVEF and preoperative LVESD were comparable, but NPV was higher for preoperative forward LVEF than for preoperative LVESD. The NPV of forward LVEF was also higher than that of LVESD, RVol, and RF. The NPV result indicated that $94.6 \%$ of patients with preoperative forward LVEF $>31.8 \%$ would truly not have postoperative LVD, suggesting that forward LVEF may be a useful parameter in the follow-up of patients with DMR and normal LVEF. Moreover, forward LVEF may be the most helpful parameter in the follow-up to detect latent LV dysfunction before mitral valve surgery in patients with DMR. Because NPV increases as disease prevalence decreases [26], caution should be exercised when interpreting the results. However, as the prevalence of LVD in our study $(22.2 \%)$ was not lower compared with that in a previous study (18\%) [7], the clinical significance of our result may not be attenuated. 
LVEF decreased more in the LVD than in the non-LVD group. LVEF is a parameter that could be affected by changes in LV preload and afterload, as well as by intrinsic LV contractility. The decrease in LVEDV (preload) from before to immediately after surgery was greater in the LVD than in the non-LVD group, whereas cESS (afterload) increased only in the LVD group. These findings may contribute, at least in part, to the greater decrease in postoperative LVEF in the LVD group. By contrast, postoperative sc-mFS, an indicator of afterload-adjusted LV contractility, was higher, despite postoperative LVEDV being lower, in the non-LVD than in the LVD group. These findings suggest that LV intrinsic contractility may be more impaired in the LVD than the non-LVD group, and that this latent LV dysfunction may be revealed postoperatively. Although postoperative LVD may be due to myocardial stunning, the latter typically resolves $48-72 \mathrm{~h}$ after ischemia. Because our patients underwent immediate postoperative echocardiographic examination which was performed at median three days (3-4 days) after surgery, myocardial stunning was an unlikely cause of postoperative LVD in the present study. This was supported by our results showing that cardiopulmonary bypass and aortic cross clamping time were not different between the two groups.

Left atrial enlargement has been reported to be a predictor of common cardiovascular outcomes such as atrial fibrillation [27]. In our data, the left atrium was enlarged in both groups, with more enlargement in the LVD group and, therefore, a higher incidence of atrial fibrillation in the LVD group than in the non-LVD group. Moreover, a larger left atrial dimeter indicates a higher left ventricular end-diastolic pressure in the LVD group than that in the non-LVD group. It has been reported that myocardial fibrosis developed in patients with MR, especially with DMR $[20,21,28]$, and the severity of myocardial fibrosis was associated with the degree of LV filling pressure [29]. Based on these previous observations, we speculate that our result on a higher incidence of atrial fibrillation in the LVD group suggests the possibility that more myocardial fibrosis may exist in the LVD group than in the non-LVD group. Further studies need to confirm this association.

Our results also showed that preoperative forward LVEF was significantly inversely correlated with RVol and RF, suggesting that forward LVEF may reflect the degree of MR, not LV systolic function. Increased RVol results in greater damage to the left ventricle, and implies a longer exposure of the left ventricle to volume overload by MR, as MR can foster a greater MR. A lower forward LVEF may reflect a greater regurgitant amount of MR, followed by more impaired LV function, which could be revealed in the absence of compensation mechanisms for overloaded volume immediately after $\mathrm{MVr}$, in MR patients with normal or even supra-normal preoperative LVEF.

In addition, we found that LVEFs at six months in the non-LVD group were still higher than in the LVD group. Compared with the LVEF immediately after surgery, the LVEF measured at six months improved in both groups, with more improvement in the LVD group than in the non-LVD group. This may, at least in part, be attributed to differences in changes in the loading conditions of the left ventricle and LV reverse remodeling after MVr. Further study of long-term trajectories of LV function using global longitudinal strain is needed in both groups to confirm this issue. We also found that three patients who experienced LVD immediately after surgery also experienced LVEF $<50 \% 6-18$ months after surgery. These findings suggest a need for additional criteria to distinguish patients who will and will not show resolution of LVD immediately after surgery. Because the number of patients was limited, however, we could not determine the significance of sustained LVEF $<50 \%$ 6-18 months after surgery.

This study had several limitations. First, some technical limitations of measuring SV using Doppler echocardiography could not be completely excluded, although experienced sonographers performed echocardiographic examination in our high-volume institution. LVOT diameter error is frequently referred to as the most common potential source of error for SV measurement by Doppler echocardiography, because it amplifies the measurement error due to the square of the LVOT diameter in continuity equation [30]. In addition, Doppler echocardiography has the limitation of underestimation of the flow 
velocity when the ultrasound beam is not parallel to the flow at LVOT. Second, this study had a retrospective observational design, with its associated inherent limitations. Third, because of limited data, we did not evaluate whether LVD occurring immediately after $\mathrm{MVr}$ was associated with a decreased LVEF 6-18 months after surgery, or with the long-term outcomes. Additional studies in larger patient cohorts are warranted.

In conclusion, the present study showed that preoperative forward LVEF with cutoff value of $31.8 \%$ could predict immediately postoperative LVD after MVr in patients with DMR and normal preoperative LVEF. Our results suggest that forward LVEF may be a useful parameter to distinguish DMR patients with and without preoperative latent LV dysfunction.

Author Contributions: J.K.: conceptualization; data curation; investigation; methodology; writingoriginal draft. J.-S.N.: data curation; investigation. Y.K.: data curation; investigation. J.-H.C.: conceptualization; methodology; data analysis; supervision; writing-review and editing. I.-C.C.: conceptualization; supervision. All authors have read and agreed to the published version of the manuscript.

Funding: This research received no external funding.

Institutional Review Board Statement: The study was conducted according to the guidelines of the Declaration of Helsinki, and approved by the Institutional Review Board of Asan Medical Center (2020-1918).

Informed Consent Statement: Patient consent was waived due to all data being acquired from a retrospective review of electronic medical records.

Data Availability Statement: The data presented in this study are available on request from the corresponding author with a reasonable reason.

Acknowledgments: The authors would like to thank to Hwa-Jung Kim, in Department of Clinical Epidemiology and Biostatistics, Asan Medical Center, for providing the professional statistical help.

Conflicts of Interest: The authors declare no conflict of interest.

\section{References}

1. d'Arcy, J.L.; Coffey, S.; Loudon, M.A.; Kennedy, A.; Pearson-Stuttard, J.; Birks, J.; Frangou, E.; Farmer, A.J.; Mant, D.; Wilson, J.; et al. Large-scale community echocardiographic screening reveals a major burden of undiagnosed valvular heart disease in older people: The OxVALVE Population Cohort Study. Eur. Heart J. 2016, 37, 3515-3522. [CrossRef]

2. Nishimura, R.A.; Otto, C.M.; Bonow, R.O.; Carabello, B.A.; Erwin, J.P.; Fleisher, L.A.; Jneid, H.; Mack, M.J.; McLeod, C.J.; O'Gara, P.T.; et al. 2017 AHA/ACC focused update of the 2014 AHA/ACC guideline for the management of patients with valvular heart disease: A report of the american college of cardiology/american heart association task force on clinical practice guidelines. Circulation 2017, 135, e1159-e1195. [CrossRef]

3. Crawford, M.H.; Souchek, J.; Oprian, C.A.; Miller, D.C.; Rahimtoola, S.; Giacomini, J.C.; Sethi, G.; Hammermeister, K.E. Determinants of survival and left ventricular performance after mitral valve replacement. Department of Veterans Affairs Cooperative Study on Valvular Heart Disease. Circulation 1990, 81, 1173-1181. [CrossRef] [PubMed]

4. Enriquez-Sarano, M.; Tajik, A.J.; Schaff, H.; Orszulak, T.A.; Bailey, K.R.; Frye, R.L. Echocardiographic prediction of survival after surgical correction of organic mitral regurgitation. Circulation 1994, 90, 830-837. [CrossRef] [PubMed]

5. Dujardin, K.S.; Seward, J.B.; Orszulak, T.A.; Schaff, H.; Bailey, K.R.; Tajik, A.J.; Enriquez-Sarano, M. Outcome after surgery for mitral regurgitation. Determinants of postoperative morbidity and mortality. J. Heart Valve Dis. 1997, 6, 17-21.

6. Suri, R.M.; Vanoverscheide, J.-L.; Grigioni, F.; Schaff, H.V.; Tribouilloy, C.; Avierinos, J.-F.; Barbieri, A.; Pasquet, A.; Huebner, M.; Rusinaru, D.; et al. Association between early surgical intervention vs watchful waiting and outcomes for mitral regurgitation due to flail mitral valve leaflets. Surv. Anesthesiol. 2014, 58, 271-272. [CrossRef]

7. Quintana, E.; Suri, R.M.; Thalji, N.M.; Daly, R.C.; Dearani, J.A.; Burkhart, H.M.; Li, Z.; Enriquez-Sarano, M.; Schaff, H.V. Left ventricular dysfunction after mitral valve repair-The fallacy of "normal" preoperative myocardial function. J. Thorac. Cardiovasc. Surg. 2014, 148, 2752-2760. [CrossRef]

8. Tribouilloy, C.; Rusinaru, D.; Szymanski, C.; Mezghani, S.; Fournier, A.; Lévy, F.; Peltier, M.; Ben Ammar, A.; Carmi, D.; Remadi, J.-P.; et al. Predicting left ventricular dysfunction after valve repair for mitral regurgitation due to leaflet prolapse: Additive value of left ventricular end-systolic dimension to ejection fraction. Eur. J. Echocardiogr. 2011, 12, 702-710. [CrossRef]

9. Enriquez-Sarano, M.; Tajik, A.; Schaff, H.; Orszulak, T.A.; McGoon, M.D.; Bailey, K.R.; Frye, R.L. Echocardiographic prediction of left ventricular function after correction of mitral regurgitation: Results and clinical implications. J. Am. Coll. Cardiol. 1994, 24, 1536-1543. [CrossRef] 
10. Matsumura, T.; Ohtaki, E.; Tanaka, K. Echocardiographic prediction of left ventricular dysfunction after mitral valve repair for mitral regurgitation as an indicator to decide the optimal timing of repair. J. Am. Coll. Cardiol. 2004, 13, 28. [CrossRef]

11. Pandis, D.; Sengupta, P.P.; Castillo, J.G.; Caracciolo, G.; Fischer, G.W.; Narula, J.; Anyanwu, A.; Adams, D.H. Assessment of longitudinal myocardial mechanics in patients with degenerative mitral valve regurgitation predicts postoperative worsening of left ventricular systolic function. J. Am. Soc. Echocardiogr. 2014, 27, 627-638. [CrossRef] [PubMed]

12. Witkowski, T.; Thomas, J.D.; Debonnaire, P.J.; Delgado, V.; Hoke, U.; Ewe, S.H.; Versteegh, M.I.; Holman, E.R.; Schalij, M.J.; Bax, J.J.; et al. Global longitudinal strain predicts left ventricular dysfunction after mitral valve repair. Eur. Heart. J. Cardiovasc. Imaging. 2013, 14, 69-76. [CrossRef]

13. Suri, R.M.; Schaff, H.; Dearani, J.A.; Sundt, T.M.; Daly, R.C.; Mullany, C.J.; Sarano, M.E.; Orszulak, T.A. Determinants of early decline in ejection fraction after surgical correction of mitral regurgitation. J. Thorac. Cardiovasc. Surg. 2008, 136, $442-447$. [CrossRef] [PubMed]

14. Dupuis, M.; Mahjoub, H.; Clavel, M.-A.; Côté, N.; Toubal, O.; Tastet, L.; Dumesnil, J.G.; O'Connor, K.; Dahou, A.; Thébault, C.; et al. Forward left ventricular ejection fraction: A simple risk marker in patients with primary mitral regurgitation. J. Am. Heart. Assoc. 2017, 6. [CrossRef] [PubMed]

15. Mitchell, C.; Rahko, P.S.; Blauwet, L.A.; Canaday, B.; Finstuen, J.A.; Foster, M.C.; Horton, K.; Ogunyankin, K.O.; Palma, R.A.; Velazquez, E.J. Guidelines for performing a comprehensive transthoracic echocardiographic examination in adults: Recommendations from the american society of echocardiography. J. Am. Soc. Echocardiogr. 2019, 32, 1-64. [CrossRef] [PubMed]

16. Zoghbi, W.A.; Adams, D.; Bonow, R.O.; Enriquez-Sarano, M.; Foster, E.; Grayburn, P.A.; Hahn, R.T.; Han, Y.; Hung, J.; Lang, R.M.; et al. Recommendations for noninvasive evaluation of native valvular regurgitation: A report from the American society of echocardiography developed in collaboration with the society for cardiovascular magnetic resonance. J. Am. Soc. Echocardiogr. 2020, 4, 58. [CrossRef]

17. Shimizu, G.; Hirota, Y.; Kita, Y.; Kawamura, K.; Saito, T.; Gaasch, W.H. Left ventricular midwall mechanics in systemic arterial hypertension. Myocardial function is depressed in pressure-overload hypertrophy. Circulation 1991, 83, 1676-1684. [CrossRef] [PubMed]

18. Gaasch, W.H.; Zile, M.; Hoshino, P.K.; Apstein, C.S.; Blaustein, A.S.; Gaasch, W.H.; Zile, M.; Hoshino, P.K.; Apstein, C.S.; Blaustein, A.S. Stress-shortening relations and myocardial blood flow in compensated and failing canine hearts with pressure-overload hypertrophy. Circulation 1989, 79, 872-883. [CrossRef]

19. de Simone, G.; Devereux, R.B.; Roman, M.J.; Ganau, A.; Saba, P.S.; Alderman, M.H.; Laragh, J.H. Assessment of left ventricular function by the midwall fractional shortening/end-systolic stress relation in human hypertension. J. Am. Coll. Cardiol. 1994, 23, 1444-1451. [CrossRef]

20. Kitkungvan, D.; Nabi, F.; Kim, R.J.; Bonow, R.O.; Khan, A.; Xu, J.; Little, S.H.; Quinones, M.A.; Lawrie, G.M.; Zoghbi, W.A.; et al. Myocardial fibrosis in patients with primary mitral regurgitation with and without prolapse. J. Am. Coll. Cardiol. 2018, 72, 823-834. [CrossRef] [PubMed]

21. Edwards, N.C.; Moody, W.E.; Yuan, M.; Weale, P.; Neal, D.; Townend, J.; Steeds, R. Quantification of left ventricular interstitial fibrosis in asymptomatic chronic primary degenerative mitral regurgitation. Circ. Cardiovasc. Imaging 2014, 7, 946-953. [CrossRef] [PubMed]

22. Ahmed, M.I.; Gladden, J.D.; Litovsky, S.H.; Lloyd, S.G.; Gupta, H.; Inusah, S.; Denney, T.; Powell, P.; McGiffin, D.C.; Dell'Italia, L.J. Increased oxidative stress and cardiomyocyte myofibrillar degeneration in patients with chronic isolated mitral regurgitation and ejection fraction > 60\%. J. Am. Coll. Cardiol. 2010, 55, 671-679. [CrossRef] [PubMed]

23. Van De Heyning, C.M.; Magne, J.; Pierard, L.A.; Bruyère, P.J.; Davin, L.; De Maeyer, C.; Paelinck, B.P.; Vrints, C.J.; Lancellotti, P. Late gadolinium enhancement CMR in primary mitral regurgitation. Eur. J. Clin. Investig. 2014, 44, 840-847. [CrossRef] [PubMed]

24. Marciniak, A.; Claus, P.; Sutherland, G.R.; Marciniak, M.; Karu, T.; Baltabaeva, A.; Merli, E.; Bijnens, B.; Jahangiri, M. Changes in systolic left ventricular function in isolated mitral regurgitation. A strain rate imaging study. Eur. Heart J. 2007, 28, 2627-2636. [CrossRef]

25. Gelfand, E.V.; Haffajee, J.A.; Hauser, T.H.; Yeon, S.B.; Goepfert, L.; Kissinger, K.V.; Delatorre, R.; Manning, W.J. Predictors of preserved left ventricular systolic function after surgery for chronic organic mitral regurgitation: A prospective study. J. Heart Valve Dis. 2010, 19, 43-50.

26. Trevethan, R. Sensitivity, specificity, and predictive values: Foundations, pliabilities, and pitfalls in research and practice. Front. Public Health 2017, 5, 307. [CrossRef]

27. Abhayaratna, W.; Seward, J.B.; Appleton, C.P.; Douglas, P.S.; Oh, J.K.; Tajik, A.J.; Tsang, T.S. Left atrial size: Physiologic determinants and clinical applications. J. Am. Coll. Cardiol. 2006, 47, 2357-2363. [CrossRef]

28. Beaufils, A.C.F.; Huttin, O.; Jobbe-Duval, A.; Senage, T.S.; Filippetti, L.; Piriou, N.; Cueff, C.; Venner, C.; Mandry, D.; Sellal, J.M.; et al. Replacement myocardial fibrosis in patients with mitral valve prolapse: Relation to mitral regurgitation, ventricular remodeling, and arrhythmia. Circulation 2021, 143, 1763-1774. [CrossRef] [PubMed]

29. Moreo, A.; Ambrosio, G.; De Chiara, B.; Pu, M.; Tran, T.; Mauri, F.; Raman, S.V. Influence of myocardial fibrosis on left ventricular diastolic function: Noninvasive assessment by cardiac magnetic resonance and echo. Circ. Cardiovasc. Imaging 2009, 2, 437-443. [CrossRef]

30. Kebed, K.; Sun, D.; Addetia, K.; Mor-Avi, V.; Markuzon, N.; Lang, R.M. Measurement errors in serial echocardiographic assessments of aortic valve stenosis severity. Int. J. Cardiovasc. Imaging 2020, 36, 471-479. [CrossRef] 